\title{
Decomposition of two functions in the orthogonality equation
}

\author{
RadosŁaW ŁukasiK AND PaWee WóJCIK
}

\begin{abstract}
The aim of this paper is to solve the orthogonality equation with two unknown functions. This problem was posed by J. Chmieliński during two international conferences.
\end{abstract}

Mathematics Subject Classification. Primary 39B52, 47A05, Secondary 47A62.

Keywords. Orthogonality equation, Hilbert space, Bounded linear operator.

\section{Introduction}

Let $\mathcal{H}, \mathcal{K}$ be Hilbert spaces over the same field $\mathbb{K} \in\{\mathbb{R}, \mathbb{C}\}$. Let $\langle\cdot \mid \cdot\rangle$ denote the inner product and $\|\cdot\|$ the norm associated with it. We shall not distinguish between the symbols used for $\mathcal{H}$ and $\mathcal{K}$. The Banach space of all bounded linear operators from $\mathcal{H}$ to $\mathcal{K}$ is denoted by $\mathcal{L}(\mathcal{H} ; \mathcal{K})$. It is known that $h: \mathcal{H} \rightarrow \mathcal{K}$ is a solution of the orthogonality equation:

$$
\forall_{x, y \in \mathcal{H}}\langle h(x) \mid h(y)\rangle=\langle x \mid y\rangle
$$

if and only if $h$ is a linear isometry (or equivalently $h \in \mathcal{L}(\mathcal{H} ; \mathcal{K})$ and $h^{*} h=I_{\mathcal{H}}$ ).

The following considerations have been inspired by the talks of J. Chmieliński during the 15th ICFEI and CUTS (see [3, p. 95] and [4, p. 145]). Namely, we will solve the generalized orthogonality equation:

$$
\forall_{x, y \in \mathcal{H}}\langle f(x) \mid g(y)\rangle=\langle x \mid y\rangle,
$$

with two unknown functions $f, g: \mathcal{H} \rightarrow \mathcal{K}$. The paper [1] also deals with Eq. (1) and similar topics. We need the following lemma for further investigations.

Lemma 1. Let $f, g: \mathcal{H} \rightarrow \mathcal{K}$ satisfy Eq. (1) and $\overline{\operatorname{Lin} f(\mathcal{H})}=\mathcal{K}$. Then $g$ is linear. Proof. Fix $y \in \operatorname{Lin} f(\mathcal{H})$. Then $y=\sum_{k=1}^{n} \alpha_{k} f\left(x_{k}\right)$ for some $\alpha_{1}, \ldots, \alpha_{n} \in \mathbb{K}$, $x_{1}, \ldots, x_{n} \in \mathcal{H}$. Thus we have 


$$
\begin{aligned}
\langle y \mid g(u+\beta w)\rangle & =\left\langle\sum_{k=1}^{n} \alpha_{k} f\left(x_{k}\right) \mid g(u+\beta w)\right\rangle=\sum_{k=1}^{n} \alpha_{k}\left\langle f\left(x_{k}\right) \mid g(u+\beta w)\right\rangle \\
& =\sum_{k=1}^{n} \alpha_{k}\left\langle x_{k} \mid u+\beta w\right\rangle=\sum_{k=1}^{n} \alpha_{k}\left(\left\langle x_{k} \mid u\right\rangle+\bar{\beta}\left\langle x_{k} \mid w\right\rangle\right) \\
& =\sum_{k=1}^{n} \alpha_{k}\left(\left\langle f\left(x_{k}\right) \mid g(u)\right\rangle+\bar{\beta}\left\langle f\left(x_{k}\right) \mid g(w)\right\rangle\right) \\
& =\sum_{k=1}^{n}\left\langle\alpha_{k} f\left(x_{k}\right) \mid g(u)+\beta g(w)\right\rangle \\
& =\left\langle\sum_{k=1}^{n} \alpha_{k} f\left(x_{k}\right) \mid g(u)+\beta g(w)\right\rangle \\
& =\langle y \mid g(u)+\beta g(w)\rangle, u, w \in \mathcal{H}, \quad \beta \in \mathbb{K} .
\end{aligned}
$$

The set $\operatorname{Lin} f(\mathcal{H})$ is dense in $\mathcal{K}$, whence $g(u+\beta w)=g(u)+\beta g(w)$ for $u, v \in \mathcal{H}$, $\beta \in \mathbb{K}$. This means that $g$ is linear.

Some well-known facts will be useful for further considerations. Let $T \in \mathcal{L}(\mathcal{K} ; \mathcal{H})$. Then there exists a unique $T^{*} \in \mathcal{L}(\mathcal{H} ; \mathcal{K})$ such that

$$
\forall_{x \in \mathcal{K}} \forall_{y \in \mathcal{H}}\langle T x \mid y\rangle_{\mathcal{H}}=\left\langle x \mid T^{*} y\right\rangle_{\mathcal{K}} .
$$

Moreover,

$$
\text { if } \forall_{x \in \mathcal{H}} \vartheta\|x\| \leqslant\|T x\|, \quad \text { then } T(\mathcal{H}) \text { is closed. }
$$

It is also known that

$T$ is invertible if and only if $T^{*}$ is invertible.

Lemma 2. Let $T_{1}, T_{2}: \mathcal{H} \rightarrow \mathcal{K}$ be linear maps and satisfy

$$
\forall_{x, y \in \mathcal{H}}\left\langle T_{1}(x) \mid T_{2}(y)\right\rangle=\langle x \mid y\rangle .
$$

Assume that $T_{1}(\mathcal{H})$ is dense. Then $T_{2}$ is continuous, i.e., $T_{2} \in \mathcal{L}(\mathcal{H} ; \mathcal{K})$.

Proof. Fix a sequence $\left(x_{n}\right)_{n=1,2, \ldots}$ such that $x_{n} \in \mathcal{H}$ and $x_{n} \rightarrow x_{o}$ for some $x_{o} \in \mathcal{H}$. Suppose that $T_{2} x_{n} \rightarrow z$ for some $z \in \mathcal{H}$. It suffices to show that $T_{2} x_{o}=z$ and apply The Closed Graph Theorem. Fix $y \in \mathcal{H}$ and notice that $\left\langle x_{n}-x_{o} \mid y\right\rangle \rightarrow 0$. On the other hand $\left\langle x_{n}-x_{o} \mid y\right\rangle \stackrel{(4)}{=}\left\langle T_{2} x_{n}-T_{2} x_{o} \mid T_{1} y\right\rangle \rightarrow$ $\left\langle z-T_{2} x_{o} \mid T_{1} y\right\rangle$. Thus we get

$$
\forall y \in \mathcal{H} \quad\left\langle z-T_{2} x_{o} \mid T_{1} y\right\rangle=0 .
$$

Since $T_{1}(\mathcal{H})$ is dense in $\mathcal{K}$, it follows that $z-T_{2} x_{o}=0$. We have shown that the graph of $T_{2}$ is closed.

Lemma 3. Let $T, S \in \mathcal{L}(\mathcal{H} ; \mathcal{K})$ satisfy the equation

$$
\forall_{x, y \in \mathcal{H}}\langle T(x) \mid S(y)\rangle=\langle x \mid y\rangle .
$$


Then $T$ is invertible if and only if $S$ is invertible.

Proof. Assume that $T$ is invertible. Then $\langle x \mid y\rangle=\langle T x \mid S y\rangle=\left\langle x \mid T^{*} S y\right\rangle$ for all $x, y \in \mathcal{H}$. Thus we have $I_{\mathcal{H}}=T^{*} S$. It follows from Lemma (3) that $T^{*}$ is invertible. Therefore $\left(T^{*}\right)^{-1}=S$, hence $S$ is invertible. The proof of the reverse is the same.

\section{Main result}

In this section, we solve functional Eq. (1). Now we can state and prove the main result of the paper.

Theorem 4. Let $f, g: \mathcal{H} \rightarrow \mathcal{K}$ satisfy Eq. (1) if and only if there exist suitable closed subspaces $\mathcal{M}_{1}, \mathcal{M}_{2}, \mathcal{M}_{3} \subset \mathcal{K}$ such that $\mathcal{K}=\mathcal{M}_{1}+\mathcal{M}_{2}+\mathcal{M}_{3}, \mathcal{M}_{k} \perp \mathcal{M}_{j}$ (for $k \neq j$ ) and $f, g$ can be written as the following decomposition

$$
f=A+\varphi, \quad g=\left(A^{*}\right)^{-1}+\gamma,
$$

for some invertible $A \in \mathcal{L}\left(\mathcal{H} ; \mathcal{M}_{1}\right)$ and for some mappings $\varphi: \mathcal{H} \rightarrow \mathcal{M}_{2}, \gamma: \mathcal{H} \rightarrow$ $\mathcal{M}_{3}$.

Proof. The implication " $\Leftarrow$ " is immediate. We start with proving " $\Rightarrow$ ". It is clear that $\mathcal{K}=\overline{\operatorname{Lin} f(\mathcal{H})} \oplus \overline{\operatorname{Lin} f(\mathcal{H})}{ }^{\perp}$, whence there are two mappings $T_{1}: \mathcal{H} \rightarrow$ $\overline{\operatorname{Lin} f(\mathcal{H})}$ and $\varphi_{1}: \mathcal{H} \rightarrow \overline{\operatorname{Lin} f(\mathcal{H})}{ }^{\perp}$ such that $g(x)=T_{1}(x)+\varphi_{1}(x)$ for all $x \in \mathcal{H}$. It is easy to check that $f, T_{1}$ satisfy

$$
\forall_{x, y \in \mathcal{H}}\left\langle f(x) \mid T_{1}(y)\right\rangle=\langle x \mid y\rangle .
$$

Indeed,

$$
\begin{aligned}
\langle x \mid y\rangle=\langle f(x) \mid g(y)\rangle= & \left\langle f(x) \mid T_{1}(y)+\varphi_{1}(y)\right\rangle=\left\langle f(x) \mid T_{1}(y)\right\rangle+\left\langle f(x) \mid \varphi_{1}(y)\right\rangle \\
& =\left\langle f(x) \mid T_{1}(y)\right\rangle+0=\left\langle f(x) \mid T_{1}(y)\right\rangle .
\end{aligned}
$$

We have shown that $f, T_{1}$ satisfy $(6)$. Moreover, the $\operatorname{set} \operatorname{Lin} f(\mathcal{H})$ is dense in $\overline{\operatorname{Lin} f(\mathcal{H})}$. It follows from 1 that $T_{1}$ is linear.

There exists the closed subspace $\mathcal{M} \subset \overline{\operatorname{Lin} f(\mathcal{H})}$ such that $\overline{T_{1}(\mathcal{H})} \perp \mathcal{M}$ and $\overline{\operatorname{Lin} f(\mathcal{H})}=\overline{T_{1}(\mathcal{H})} \oplus \mathcal{M}$. Therefore there are two mappings $T_{2}: \mathcal{H} \rightarrow \overline{T_{1}(\mathcal{H})}$ and $\varphi_{2}: \mathcal{H} \rightarrow \mathcal{M}$ such that $f(x)=T_{2}(x)+\varphi_{2}(x)$ for all $x \in \mathcal{H}$. In a similar way we prove that $T_{2}, T_{1}$ satisfy

$$
\forall_{x, y \in \mathcal{H}} \quad\left\langle T_{2}(x) \mid T_{1}(y)\right\rangle=\langle x \mid y\rangle .
$$

Now, we can consider the linear operators $T_{2}: \mathcal{H} \rightarrow \overline{T_{1}(\mathcal{H})}$ and $T_{1}: \mathcal{H} \rightarrow \overline{T_{1}(\mathcal{H})}$ instead of $T_{1}: \mathcal{H} \rightarrow \overline{\operatorname{Lin} f(\mathcal{H})}$. By Lemma 1 and $(7), T_{2}$ is linear. By Lemma 2 and $(7), T_{2}$ is continuous, i.e., $T_{2} \in \mathcal{L}\left(\mathcal{H} ; \overline{T_{1}(\mathcal{H})}\right)$.

There is the closed subspace $\mathcal{N} \subset \overline{T_{1}(\mathcal{H})}$ such that $\overline{T_{1}(\mathcal{H})}=\overline{T_{2}(\mathcal{H})} \oplus \mathcal{N}$ and $T_{2}(\mathcal{H}) \perp \mathcal{N}$. Hence there are two mappings $T_{3}: \mathcal{H} \rightarrow \overline{T_{2}(\mathcal{H})}$ and $\varphi_{3}: \mathcal{H} \rightarrow \mathcal{N}$ such 
that $T_{1}(x)=T_{3}(x)+\varphi_{3}(x)$ for all $x \in \mathcal{H}$. In a similar way we prove that $T_{3}$ is linear and $T_{2}, T_{3}$ satisfy

$$
\forall_{x, y \in \mathcal{H}} \quad\left\langle T_{2}(x) \mid T_{3}(y)\right\rangle=\langle x \mid y\rangle .
$$

Now, we consider the linear operator $T_{2} \in \mathcal{L}\left(\mathcal{H} ; \overline{T_{2}(\mathcal{H})}\right.$ ) (instead of $T_{2} \in \mathcal{L}\left(\mathcal{H} ; \overline{T_{1}(\mathcal{H})}\right)$ ). Let us consider also the linear operator $T_{3}: \mathcal{H} \rightarrow \overline{T_{2}(\mathcal{H})}$. Applying again Lemma 2 and (8) we can say that $T_{3}$ is continuous, i.e., $T_{3} \in \mathcal{L}\left(\mathcal{H} ; \overline{T_{2}(\mathcal{H})}\right)$. Now, we get

$$
\|x\|^{2}=\langle x \mid x\rangle \stackrel{(8)}{=}\left\langle T_{2} x \mid T_{3} x\right\rangle \leqslant\left\|T_{2} x\right\| \cdot\left\|T_{3} x\right\| \leqslant\left\|T_{2} x\right\| \cdot\left\|T_{3}\right\| \cdot\|x\|
$$

for all $x \in \mathcal{H}$. It follows from the above inequality that

$$
\forall y \in \mathcal{H} \frac{1}{\left\|T_{3}\right\|} \cdot\|x\| \leqslant\left\|T_{2} x\right\| .
$$

Thus $T_{2}(\mathcal{H})$ is closed (see 2). Thus we obtain that $T_{2}(\mathcal{H})=\overline{T_{2}(\mathcal{H})}$ and $T_{2}$, $T_{3} \in \mathcal{L}\left(\mathcal{H} ; T_{2}(\mathcal{H})\right)$. It follows from $(9)$ that $T_{2}$ in injective, hence $T_{2}$ is invertible. Therefore $T_{3} \in \mathcal{L}\left(\mathcal{H} ; T_{2}(\mathcal{H})\right)$ is also invertible by Theorem 3 and (8).

Finally, we define $\mathcal{M}_{1}:=T_{2}(\mathcal{H}), \mathcal{M}_{2}:=\mathcal{M}$ and $\mathcal{M}_{3}:=\mathcal{N} \oplus \overline{\operatorname{Lin} f(\mathcal{H})}{ }^{\perp}$. Next, we define $\varphi: \mathcal{H} \rightarrow \mathcal{M}_{2}, \gamma: \mathcal{H} \rightarrow \mathcal{M}_{3}$ by $\varphi:=\varphi_{2}, \gamma:=\varphi_{3}+\varphi_{1}$. Moreover, we define $A \in \mathcal{L}\left(\mathcal{H} ; \mathcal{M}_{1}\right)$ by $A:=T_{2}$. Clearly $A$ is invertable and $\left(A^{*}\right)^{-1}=T_{3}$. Thus we get $f=A+\varphi$ and $g=\left(A^{*}\right)^{-1}+\gamma$ and $\mathcal{K}=\mathcal{M}_{1}+\mathcal{M}_{2}+\mathcal{M}_{3}$, where $\mathcal{M}_{k} \perp \mathcal{M}_{j}$ for $k \neq j$.

Corollary 5. Suppose that $\operatorname{dim} \mathcal{H}<\infty$. Let $f, g: \mathcal{H} \rightarrow \mathcal{H}$ satisfy Eq. (1). Then $f, g \in \mathcal{L}(\mathcal{H} ; \mathcal{H})$ and $f=\left(g^{*}\right)^{-1}$.

Proof. By the above theorem we have a decomposition $f=A+\varphi, g=\left(A^{*}\right)^{-1}+\gamma$ for some invertible $A \in \mathcal{L}\left(\mathcal{H} ; \mathcal{M}_{1}\right)$ and some mappings $\varphi: \mathcal{H} \rightarrow \mathcal{M}_{2}, \gamma: \mathcal{H} \rightarrow \mathcal{M}_{3}$ (where $\mathcal{H}=\mathcal{M}_{1}+\mathcal{M}_{2}+\mathcal{M}_{3}$ and $\mathcal{M}_{k} \perp \mathcal{M}_{j}$ ). Since $A \in \mathcal{L}\left(\mathcal{H} ; \mathcal{M}_{1}\right)$ is invertible and $\operatorname{dim} \mathcal{H}<\infty$, it follows that $\mathcal{M}_{1}=\mathcal{H}$ and $\mathcal{M}_{2}=\{0\}=\mathcal{M}_{3}$. So $\varphi=0=$ $\gamma$.

Corollary 6. Let $f: \mathcal{H} \rightarrow \mathcal{K}$ satisfy the equation

$$
\forall_{x, y \in \mathcal{H}}\langle f(x) \mid f(y)\rangle=\langle x \mid y\rangle .
$$

Then $f$ is a linear isometry.

Proof. By Theorem 4 we have a decomposition $f=A+\varphi, f=\left(A^{*}\right)^{-1}+\gamma$ for some $A \in \mathcal{L}\left(\mathcal{H} ; \mathcal{M}_{1}\right)$ and $\varphi: \mathcal{H} \rightarrow \mathcal{M}_{2}, \gamma: \mathcal{H} \rightarrow \mathcal{M}_{3}$. Thus we get $A+\varphi=\left(A^{*}\right)^{-1}+\gamma$. Since $\mathcal{M}_{2} \perp \mathcal{M}_{3}$, we get $\varphi=0=\gamma$. Hence $f=A$. Then $f$ is a linear mapping and by (10), $f$ is a linear isometry.

Similar investigations have been carried out in the manuscript [2]. Namely, Eq. (1) and its stability, as well as the approximate orthogonality preserving property are considered in [2]. 
Open Access. This article is distributed under the terms of the Creative Commons Attribution 4.0 International License (http://creativecommons.org/licenses/by/4.0/), which permits unrestricted use, distribution, and reproduction in any medium, provided you give appropriate credit to the original author(s) and the source, provide a link to the Creative Commons license, and indicate if changes were made.

\section{References}

[1] Chmieliński, J.: Orthogonality equation with two unknown functions. Aequationes Math., to appear; doi:10.1007/s00010-015-0359-x

[2] Chmieliński, J., Łukasik, R., Wójcik, P.: On the stability of the orthogonality equation and orthogonality preserving property with two unknown functions (submitted)

[3] Report of Meeting, Annales Universitatis Paedagogicae Cracoviensis Studia Mathematica, Vol. 12, pp. 91-131 (2013)

[4] Report of Meeting, Annales Universitatis Paedagogicae Cracoviensis Studia Mathematica, Vol. 13, pp. 139-169 (2014)

Radosław Eukasik

Institute of Mathematics

University of Silesia

ul. Bankowa 14, 40-007 Katowice, Poland

e-mail: rlukasik@math.us.edu.pl

Paweł Wójcik

Institute of Mathematics

Pedagogical University of Cracow

Podchorążych 2, 30-084 Kraków, Poland

e-mail: pwojcik@up.krakow.pl

Received: January 25, 2015

Revised: September 30, 2015 